A sensitivity analysis similar to 2 , but based on the casecohort study of lung cancer included in the NCI cohort study.

A pooled analysis of individual-level data from the two existing US historical cohort mortality studies of AN-exposed workers, the NCI cohort and the DuPont Company cohort of two AN production sites.

Results We will report current progress and available results from our series of investigations.

\section{PRELIMINARY RESULTS OF A CASE-CONTROL STUDY OF NIGHT SHIFT WORK AND BREAST CANCER AMONG HONG KONG WOMEN}

${ }^{1}$ Tse, ${ }^{1}$ Wang, ${ }^{2}$ Chan, ${ }^{3}$ Kwok, ${ }^{4}$ Leung, ${ }^{1} Y u .{ }^{1}$ The Chinese University of Hong Kong, NT, Hongkong; 'Breast Surgery NTEC Hospital Authority, North District Hospital, Hong Kong SAR, Hongkong; ${ }^{3}$ Department of Oncology, Princess Margaret Hospital, Hong Kong SAR, Hongkong; ${ }^{4}$ Department of Surgery, Yan Chai Hospital, Hong Kong $S A R$, Hongkong

\subsection{6/oemed-2013-101717.160}

Objectives Breast cancer is the leading cause of cancer morbidity and mortality among women in Hong Kong, and the rate is accelerating. Meanwhile, the prevalence of night shift work in Hong Kong women is also increasing. We aim to present the preliminary results of an ongoing case-control study of breast cancer among Hong Kong women in the EPI$\mathrm{COH}$ 2.0.13.

Methods We are consecutively recruiting all newly diagnosed breast cancer cases and age-matched controls from several hospitals and we expect to collect 1,066 cases and 1,066 controls by the end of 2013. A standardised questionnaire was used to collect information on each participant's lifetime exposure to night shift work, exposure to light at night, sleep disorders, environmental exposures to pesticides and other EDCs, occupational exposures, reproductive and anthropometric factors, smoking, diet, alcohol drinking, family cancer history, etc.

Results We have obtained 350 breast cancer cases and 350 controls with a response rate of $92 \%$. The age distribution at the diagnosis of breast cancer $(55.1 \pm 11.9$ vs. $54.2 \pm 14.6, \mathrm{p}=$ $0.39)$ and the menopause status $(61.9 \%$ vs. $61.0 \%, \mathrm{p}=0.84)$ for the cases and controls are comparable. A slightly more controls $(92.2 \%)$ than the cases $(89.9 \%)$ are the never smokers. Around $80 \%$ cases have records of estrogen receptor (ER) status and $73.3 \%$ of them are ER positive, while $52 \%$ are positive in progesterone receptor. The proportion of ever exposure of night shift work tends to be low (10\%) and this proportion is not significantly higher in cases.

Conclusions This study showed preliminary results regarding the basic characteristics of an ongoing case-control study of breast cancer in Hong Kong women. In addition to patient interview, we have also collected blood samples. We shall be able to present more valuable data in the upcoming EPICOH.

Acknowledgement Research Grants Council, Hong Kong (Project no. 474811).

\section{NIGHT-SHIFT WORK AND CHRONIC LYMPHOCYTIC LEUKAEMIA RISK}

1'L C Costas, ${ }^{2}$ Papantoniou, ${ }^{3}$ Tardón, ${ }^{4}$ García-Martín, 5 Javier, ${ }^{1}$ de Sanjosé. ${ }^{1}$ Catalan Institute of Oncology, Barcelona, Spain; ${ }^{2}$ Centre for Research in Environmental Epidemiology, Barcelona, Spain; ${ }^{3}$ Universidad de Oviedo, Oviedo, Spain; ${ }^{4}$ Universidad de Granada, Granada, Spain; ${ }^{5}$ Universidad de Cantabria, Santander, Spain

10.1136/oemed-2013-101717.161
Objectives Chronic lymphocytic leukaemia (CLL) prognosis has been related to several alterations in the circadian molecular signalling. Other evidences suggest that night work may increase mature B-cell neoplasms risk. Although CLL was considered as an independent diagnostic entity for about a century, it is currently considered a mature B-cell neoplasm subtype. However, no epidemiologic data exists in regard to night work and CLL risk to date. We aim to determine if night work is a risk factor for CLL.

Methods We evaluated 521 cases and 1,511 controls in four areas of Spain within the population-based multi-case-control study MCC-Spain (www.mccspain.org) in collaboration with the International Cancer Genome Consortium of CLL (www.icgc. org). Participants were interviewed face-to-face by trained interviewers for information on socio-demographic factors, reproductive, familial, medical and occupational history, and other lifestyle factors. The occupational section included questions for each job that was held for one year or longer, including shifts, years of debut and end, and amount of hours worked each day. We used logistic regression adjusting for potential confounders.

Results 62 cases $(12 \%)$ and 154 controls $(10 \%)$ had at least one permanent night job, and 48 cases (9\%) and 178 controls (12\%) had worked in a job with four or more nights/month and/or with $20 \%$ of the work performed by night. None of these two categories was associated to LLC risk $(\mathrm{OR}=0.9595 \% \mathrm{CI}=$ 0.68 to 1.34 for permanent night and $\mathrm{OR}=0.7995 \% \mathrm{CI}=$ 0.55 to 1.15 for rotating night shifts compared to day work, respectively). ORs were higher among subjects working more than 5 years in permanent night shift, but results were not significant.

Conclusions Our data suggest that night work does not play a significant role in CLL aetiology. The interpretation of these results may be hampered by the low sample size exposed to long term night work.

\section{USING CARCINOGENIC CLASSIFICATIONS OF PESTICIDES TO EVALUATE THE RISK OF SELECT CANCERS IN CANADIAN MEN}

${ }^{1} \mathrm{M}$ P Pahwa, ${ }^{1}$ Demers, ${ }^{1}$ Kachuri, ${ }^{1}$ Navaranjan, ${ }^{2}$ Blair, ${ }^{1}$ Hohenadel, ${ }^{3}$ Spinelli, ${ }^{4}$ McLaughlin, ${ }^{5}$ Dosman, ${ }^{5}$ Pahwa, ${ }^{6}$ Harris. ${ }^{1}$ Occupational Cancer Research Centre, Toronto, Canada; ${ }^{2}$ United States National Cancer Institute, Bethesda, United States of America; ${ }^{3}$ British Columbia Cancer Agency Research Centre, Vancouver, Canada; ${ }^{4}$ Samuel Lunenfeld Research Institute, Toronto, Canada; ${ }^{5}$ Canadian Centre for Health and Safety in Agriculture, University of Saskatchewan, Saskatoon, Canada; ${ }^{6}$ Cancer Care Ontario, Toronto, Canada

\subsection{6/oemed-2013-101717.162}

Objective To examine possible associations between exposure to pesticides classified by their carcinogenicity and the risk of select cancers in Canadian men.

Methods Between 1991 and 1994, data were collected in six provinces using paper and telephone questionnaires from cases with incident non-Hodgkin lymphoma (NHL) $(\mathrm{N}=513)$, multiple myeloma $(\mathrm{MM})(\mathrm{N}=342)$, soft tissue sarcoma (STS) $(\mathrm{N}=$ $357)$, and Hodgkin lymphoma (HL) $(\mathrm{N}=316)$ and a random, population-based sample of 1506 age- and province-matched controls. Pesticides were grouped into carcinogenic categories using a composite score created from evaluations by the International Agency for Research on Cancer (IARC) and US Environmental Protection Agency (US EPA). Pesticides were categorised as "probably" carcinogenic (IARC Group 2A and/or US EPA Group B and higher) or "possibly" carcinogenic (IARC Group 